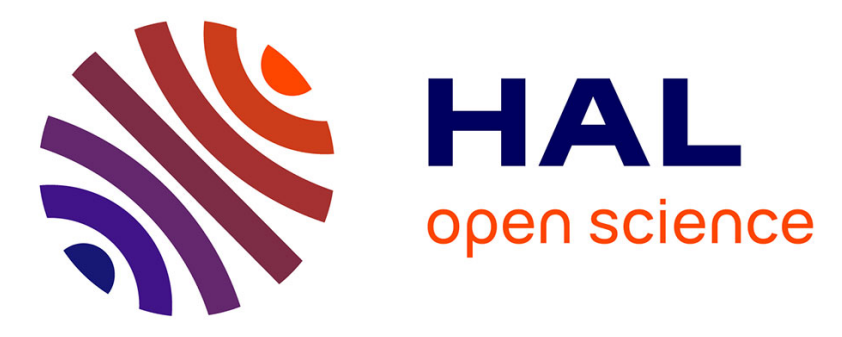

\title{
Power-Splitting Protocol in Power Beacon-assisted Energy Harvesting Full-Duplex Relaying Networks: Performance Analysis
}

Tan Nguyen, Phu Tran Tin, Phuong T. Tran, Tran Hoang Quang Minh, Miroslav Voznak

\section{To cite this version:}

Tan Nguyen, Phu Tran Tin, Phuong T. Tran, Tran Hoang Quang Minh, Miroslav Voznak. PowerSplitting Protocol in Power Beacon-assisted Energy Harvesting Full-Duplex Relaying Networks: Performance Analysis. 11th IFIP Wireless and Mobile Networking Conference (WMNC 2018), Sep 2018, Prague, Czech Republic. pp.109-115. hal-01995495

\section{HAL Id: hal-01995495 \\ https://hal.inria.fr/hal-01995495}

Submitted on 26 Jan 2019

HAL is a multi-disciplinary open access archive for the deposit and dissemination of scientific research documents, whether they are published or not. The documents may come from teaching and research institutions in France or abroad, or from public or private research centers.
L'archive ouverte pluridisciplinaire HAL, est destinée au dépôt et à la diffusion de documents scientifiques de niveau recherche, publiés ou non, émanant des établissements d'enseignement et de recherche français ou étrangers, des laboratoires publics ou privés. 


\section{Power-Splitting Protocol in Power Beacon-assisted Energy Harvesting Full-Duplex Relaying Networks: Performance Analysis}

\author{
Tan N. Nguyen \\ Faculty of Electrical Engineering and Computer Science \\ VSB-Technical University of Ostrava \\ 17. listopadu 15/2172, 70833 Ostrava, Czech Republic \\ tan.nhat.nguyen.st@,vsb.cz
}

Phu Tran Tin

Faculty of Electrical Engineering and Computer Science VSB-Technical University of Ostrava

17. listopadu 15/2172, 70833 Ostrava, Czech Republic tin.tran.phu.st@,vsb.cz

Phuong T. Tran

Faculty of Electrical \& Electronics Engineering Ton Duc Thang University

19 Nguyen Huu Tho Street, Ho Chi Minh City, Vietnam tranthanhphuong@,tdt.edu.vn

\begin{abstract}
In this paper, we investigate the full-duplex power beacon-assisted energy harvesting relay network in the power splitting protocol. The proposed system model consists of a Source node (S), a Relay node (R), a Destination node (D) and a Power-assisted beacon node (PB). In this model, $S$ first harvests energy from $P B$ in the downlink (DL) and then uses the harvested energy to transmit information to $D$ via helping $R$ in the power splitting protocol. Firstly, the analytical expressions of the system performance are proposed and derived. After that, the analytical results are also demonstrated and convinced by using Monte-Carlo simulation. The numerical results demonstrated and convinced the analytical and the simulation results are matched well with each other in connection with all system parameters.
\end{abstract}

Keywords - Full-duplex, Power-assisted beacon (PB) throughput, outage probability, wireless energy harvesting (EH).

\section{INTRODUCTION}

Energy harvesting relay network, which uses a radio frequency (RF) signal for wireless power transfer, has attracted much attention because of prolonging the lifetime of a wireless network [1-5]. This solution based on the fact that RF signal can carry both energy and information simultaneously and replace or recharge batteries incurs a high cost and can be inconvenient or hazardous (e.g., in toxic environments), or highly undesirable (e.g., for sensors embedded in building structures or inside the human body) [6]. In the last decade, the

\author{
Tran Hoang Quang Minh (Corresponding Author) \\ Optoelectronics Research Group, Faculty of Electrical and \\ Electronics Engineering \\ Ton Duc Thang University \\ Ho Chi Minh City, Vietnam \\ tranhoangquangminh@,tdt.edu.vn
}

\author{
Miroslav Voznak \\ Faculty of Electrical Engineering and Computer Science \\ VSB-Technical University of Ostrava \\ 17. listopadu 15/2172, 70833 Ostrava, Czech Republic \\ miroslav.voznak@vvb.cz
}

system performance of the energy harvesting relay network has been considered in many studies. The paper [7] investigated the full-duplex energy harvesting relay network with the simultaneous energy harvesting and information transmission. Moreover, the development of cooperative protocols for energy harvesting relay network is deeply studied in [8-9]. Authors in [10-11] dealt with using bio-inspired algorithms for energy harvesting. Furthermore, [12-13] proposed a "harvest-thentransmit" protocol for a multi-user relay network. In all papers above, the energy source of the whole network is only the source or the access point. In the trends to improving the energy harvesting and information transmission processes in the wireless relay network, some researchers proposed the idea of deploying dedicated power beacon node (PB). In the wireless relay network with using the $\mathrm{PB}$ node, the destination (D) can harvest wireless energy not only from the source (S) node but also from the deployed PB node [13-14]. For this new model, the question of system performance is still open and is necessary to investigate, and this is the aim of our paper. In this research, we propose and investigate the system performance analysis of the full-duplex power beacon-assisted energy harvesting relay network in the power splitting protocol. All the transmission channels are the Raleigh environment with random distribution signal. The main contributions of the paper are summarized as follows:

1) Propose the system model of the full-duplex power beacon-assisted energy harvesting relay network with the power splitting protocol. 
2) Derive the integral closed-form expressions of the outage probability and throughput of the proposed system.

3) The optimal power splitting factor of the proposed system is demonstrated entirely.

The rest of this paper is organized as follows. We first propose the system model in Section II. Then, the system performance analysis is investigated and derived in section III. In section IV, some numerical results and discussions are proposed. Finally, Section V concludes the paper.

\section{SYSTEM MODEL}

In this section, the full-duplex power beacon-assisted energy harvesting relay network with the power splitting protocol is illustrated in Fig. 1. In Fig. 1a, the information is transferred from the source node (S) to the destination node (D) with helping of an intermediate relay $(\mathrm{R})$. The $\mathrm{S}$ has received energy from the $\mathrm{PB}$ node directly. We assume that all the block-fading channels between the PB, S, R, and D are the Raleigh fading channels. Fig. 1b displayed the power splitting based protocol model. In the first half interval $T / 2$, the $S$ harvests energy from the PB node. After that, the energy transfer from the $\mathrm{S}$ to the $\mathrm{R}$ and the information transmission from the $\mathrm{S}$ to the $\mathrm{D}$ via $\mathrm{R}$ in the second half interval time $\mathrm{T} / 2,0$ $\leq \rho \leq 1[18]$.

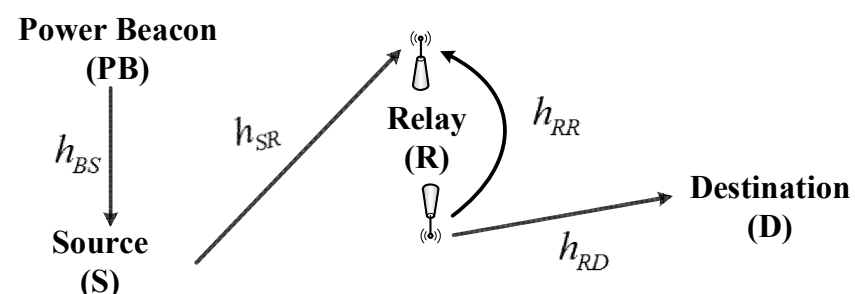

(S)

(a)

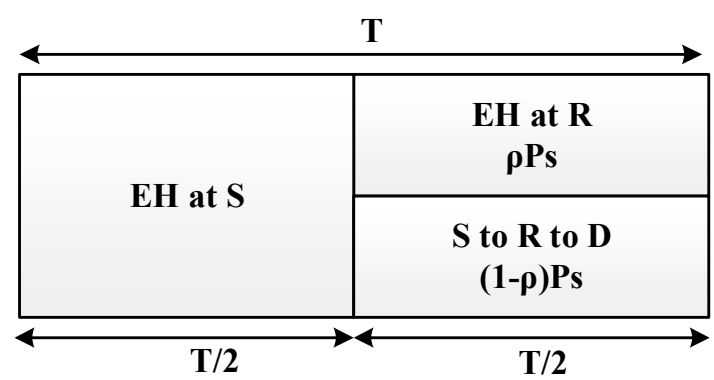

(b)

Fig. 1. The proposed system model

\section{THE SYSTEM PERFORMANCE}

In this paper, we consider a relaying network system, where each terminal operates in full-duplex mode. The two nodes $\mathrm{S}$ and D communicate with each other via the help of relay $R$ over Rayleigh fading channels. There is no direct path between $S$ and $D$. The received signal at the relay $(R)$ can be formulated as:

$$
y_{r}=\sqrt{1-\rho} h_{s r} x_{s}+h_{r r} x_{r}+n_{r}
$$

where $h_{r r}$ is loopback interference channel, $h_{s r}$ is $\mathrm{S}$ to $\mathrm{R}$ gain channel, $0<\rho<1$ is power splitting factor. The received signal at the destination can expressed as:

$$
y_{d}=h_{r d} x_{r}+n_{d}
$$

where $\mathrm{n}_{\mathrm{r}}, \mathrm{n}_{\mathrm{d}}$ : the additive white Gaussian noise (AWGN) at $R$, $D$ with zero mean and variance $\mathrm{N}_{0}$ and $h_{r d}$ is $\mathrm{R}$ to $\mathrm{D}$ gain channel. In these equations, we assume $\mathrm{E}\left[\left|x_{s}\right|^{2}\right]=P_{s}$, $\mathrm{E}\left[\left|x_{r}\right|^{2}\right]=P_{r}$, and $\mathrm{E}[\bullet]$ is expectation operator. Moreover, the harvested energy at the source can be given by the following:

$$
E_{s}=\eta_{b} P_{B}\left|h_{b s}\right|^{2}(T / 2)
$$

where $0<\eta_{b}<1$ is energy conversion efficiency at the PB node. Moreover, the average transmits power at the source can be calculated as:

$$
P_{s}=\frac{E_{s}}{T / 2}=\eta_{b} P_{B}\left|h_{b s}\right|^{2}
$$

After harvesting the energy from PB node, the S node will split the power into 2 parts:

1) $\rho P_{s}$ is used for energy harvesting at the relay node.

2) $(1-\rho) P_{s}$ is used for the information transmission from the $\mathrm{S}$ to $\mathrm{R}$. Therefore, the harvested energy at $\mathrm{R}$ can be given by the following equation:

$$
E_{r}=\eta_{s} \rho(T / 2) P_{s}\left|h_{s r}\right|^{2}
$$

where $0<\eta_{s}<1$ is energy conversion efficiency at the source node. Then the average transmits power at the relay is:

$$
P_{r}=\frac{E_{r}}{T / 2}=\eta_{s} \rho P_{s}\left|h_{s r}\right|^{2}
$$

Combine (6) with (4) we have (7):

$$
P_{r}=\eta_{s} \rho P_{s}\left|h_{s r}\right|^{2}=\eta_{s} \eta_{b} \rho P_{B}\left|h_{b s}\right|^{2}\left|h_{s r}\right|^{2}
$$

From (1) we have the signal to noise ratio (SNR) at the relay as the below equation: 


$$
\gamma_{1}=\frac{\mathrm{E}\left\{\mid \text { signal }\left.\right|^{2}\right\}}{\mathrm{E}\left\{\mid \text { noise }\left.\right|^{2}\right\}}=\frac{(1-\rho)\left|h_{s r}\right|^{2} P_{s}}{\left|h_{r r}\right|^{2} P_{r}+N_{0}}
$$

Substitution (4), (7) into (8) we can obtain (9):

$$
\begin{aligned}
\gamma_{1} & =\frac{(1-\rho)\left|h_{s r}\right|^{2} \eta_{b} P_{B}\left|h_{b s}\right|^{2}}{\left|h_{r r}\right|^{2} \eta_{s} \eta_{b} \rho P_{B}\left|h_{b s}\right|^{2}\left|h_{s r}\right|^{2}+N_{0}} \\
& =\frac{(1-\rho) \eta_{b} \gamma_{0}\left|h_{s r}\right|^{2}\left|h_{b s}\right|^{2}}{\left|h_{b s}\right|^{2}\left|h_{s r}\right|^{2}\left|h_{r r}\right|^{2} \eta_{s} \eta_{b} \rho \gamma_{0}+1}
\end{aligned}
$$

where $\gamma_{0}=\frac{P_{B}}{N_{0}}$. We assume that $\eta_{b}=\eta_{s}=\eta$ and denote $X=\left|h_{s r}\right|^{2}\left|h_{b s}\right|^{2}, Y=\left|h_{r r}\right|^{2}$. Equation (9) can be rewritten as the following:

$$
\gamma_{1}=\frac{(1-\rho) \eta \gamma_{0} X}{X Y \eta^{2} \rho \gamma_{0}+1}
$$

At the high SNR, it is mean $\gamma_{0} \rightarrow \infty$. Equation (8) can be rewritten as:

$$
\gamma_{1}=\frac{(1-\rho) \eta \gamma_{0} X}{X Y \eta^{2} \rho \gamma_{0}+1} \approx \frac{(1-\rho)}{\rho Y \eta}
$$

From(2) the signal to noise ratio at the destination can be expressed as:

$$
\gamma_{2}=\frac{P_{r}\left|h_{r d}\right|^{2}}{N_{0}}=\eta^{2} \rho \gamma_{0}\left|h_{b s}\right|^{2}\left|h_{s r}\right|^{2}\left|h_{r d}\right|^{2}=\eta^{2} \rho \gamma_{0} X Z
$$

where $Z=\left|h_{r d}\right|^{2}$. For the decode and forward mode, the end to end signal to noise ratio (SNR) can be obtained by the following equation:

$$
\gamma_{e 2 e}=\min \left(\gamma_{1}, \gamma_{2}\right)
$$

Remark 1. In this section, $h_{s r}, h_{r d}, h_{b s}, h_{r r}$ are the Rayleigh fading channels, so the probability density function (PDF) of $\left|h_{i}\right|^{2}$ can be given by (14):

$$
f_{\left|h_{i}\right|^{2}}(x)=\lambda_{i} e^{-\lambda_{h_{i}} x}
$$

where $i \in(s r, r d, b s, r r)$. Moreover, the cumulative distribution function (CDF) of $\left|h_{i}\right|^{2}$ also can be obtained by (15):

$$
F_{\left|h_{i}\right|}(x)=1-e^{-\lambda_{i} x}
$$

where $\lambda_{i}$ is the mean value of the exponential random variable $\left|h_{i}\right|^{2}$.

\section{Remark 2.}

$$
F_{X}(x)=\int_{0}^{\infty} F_{\left|h_{s r}\right|^{2}}\left(\left.\frac{x}{\left|h_{r d}\right|^{2}}|| h_{r d}\right|^{2}\right) f_{\left|h_{r d}\right|^{2}}(x) d x
$$

Utilizing the result in [15], the CDF of X can be shown as the below equation:

$$
F_{X}(x)=1-2 \sqrt{\lambda_{s r} \lambda_{b s} x} K_{1}\left(2 \sqrt{\lambda_{s r} \lambda_{b s} x}\right)
$$

where $K_{v}(\bullet)$ is the modified Bessel function of the second kind and $\mathrm{v}^{\text {th }}$ order.

\section{The outage probability:}

The Outage Probability (OP) of the proposed system can be calculated by (18) and (19):

$$
\begin{aligned}
& O P=\operatorname{Pr}\left(\gamma_{e 2 e}<\gamma_{t h}\right)=\operatorname{Pr}\left[\min \left(\gamma_{1}, \gamma_{2}\right)<\gamma_{t h}\right] \\
& \operatorname{Pr}\left\{\min \left(\frac{(1-\rho)}{\rho Y \eta}, \eta^{2} \rho \gamma_{0} X Z\right)<\gamma_{t h}\right\} \\
& =1-\operatorname{Pr}\left[\frac{(1-\rho)}{\rho Y \eta} \geq \gamma_{t h}\right] \times \operatorname{Pr}\left(\eta^{2} \rho \gamma_{0} X Z \geq \gamma_{t h}\right)
\end{aligned}
$$

where $\gamma_{t h}=2^{R}-1$, and $\mathrm{R}$ is the source rate. In this analysis, we consider

$$
\begin{aligned}
P_{1} & =\operatorname{Pr}\left[\frac{(1-\rho)}{\rho Y \eta} \geq \gamma_{t h}\right] \\
& =\operatorname{Pr}\left[Y \leq \frac{(1-\rho)}{\rho \eta \gamma_{t h}}\right]=F_{Y}\left[\frac{(1-\rho)}{\rho \eta \gamma_{t h}}\right]
\end{aligned}
$$

From (15) $\mathrm{P}_{1}$ can be obtained as:

$$
P_{1}=1-\exp \left[\frac{-\lambda_{r r}(1-\rho)}{\rho \eta \gamma_{t h}}\right]
$$

Similarly, we have: 


$$
\begin{aligned}
& P_{2}=\operatorname{Pr}\left(\eta^{2} \rho \gamma_{0} X Z \geq \gamma_{t h}\right) \\
& =1-\operatorname{Pr}\left(X<\frac{\gamma_{t h}}{\eta^{2} \rho \gamma_{0} Z}\right)=1-\int_{0}^{\infty} F_{X}\left(\frac{\gamma_{t h}}{\eta^{2} \rho \gamma_{0} Z}\right) f_{Z} d z
\end{aligned}
$$

Combine with (16), we can obtain following equation:

$$
\begin{gathered}
P_{2}=1-\int_{0}^{\infty}\left[1-2 \sqrt{\frac{\lambda_{s r} \lambda_{b s} \gamma_{t h}}{\eta^{2} \rho \gamma_{0} Z}} K_{1}\left(2 \sqrt{\frac{\lambda_{s r} \lambda_{b s} \gamma_{t h}}{\eta^{2} \rho \gamma_{0} Z}}\right)\right] f_{Z} d z \\
P_{2}=2 \lambda_{r d} \int_{0}^{\infty} \sqrt{\frac{\lambda_{s r} \lambda_{b s} \gamma_{t h}}{\eta^{2} \rho \gamma_{0} Z}} K_{1}\left(2 \sqrt{\frac{\lambda_{s r} \lambda_{b s} \gamma_{t h}}{\eta^{2} \rho \gamma_{0} Z}}\right) e^{-\lambda_{r d} z} d z \\
O P=1-2 \lambda_{r d}\left\{1-\exp \left[\frac{-\lambda_{r r}(1-\rho)}{\rho \eta \gamma_{t h}}\right]\right\} \\
\quad \int_{0}^{\infty} \sqrt{\frac{\lambda_{s r} \lambda_{b s} \gamma_{t h}}{\eta^{2} \rho \gamma_{0} Z}} K_{1}\left(2 \sqrt{\frac{\lambda s r}{\eta^{2} \rho \gamma_{0} Z}}\right) e^{-\lambda_{r d} z} d z
\end{gathered}
$$

\section{Average Throughput:}

$$
\begin{aligned}
& R_{D L}=(1-O P) \times \frac{R}{T} \times(T / 2)=(1-O P) \times \frac{R}{2} \\
& =R \lambda_{r d}\left\{1-\exp \left[\frac{-\lambda_{r r}(1-\rho)}{\rho \eta \gamma_{t h}}\right]\right\} \\
& \int_{0}^{\infty} \sqrt{\frac{\lambda_{s r} \lambda_{b s} \gamma_{t h}}{\eta^{2} \rho \gamma_{0} Z}} K_{1}\left(2 \sqrt{\frac{\lambda_{s r} \lambda_{b s} \gamma_{t h}}{\eta^{2} \rho \gamma_{0} Z}}\right) e^{-\lambda_{r d} z} d z
\end{aligned}
$$

\section{Optimal Power Splitting Factor:}

The optimal value $\rho^{*}$ can be obtained by solving the equation $\frac{d R_{D L}(\rho)}{d \rho}=0$. Given the achievable throughput expression in (26), this optimization problem does not admit a closed-form solution. However, the optimal $\rho^{*}$ is efficiently solved via numerical calculation, as illustrated below.

Here, we can use Golden section search algorithm to find the optimal factor $\rho^{*}$. This algorithm has been used in many global optimization problems in communications, for example, in [16]. The detailed algorithm, as well as the related theory, is described in [17].

\section{NUMERICAL RESULTS AND DISCUSSION}

In this section, some simulation results are presented to investigate the system performances of the full-duplex power beacon-assisted energy harvesting relay network with the power splitting protocol. Both in theoretical and Monte Carlo simulation results evaluate the system performance analysis. Also, the system performance is analyzed in connection with $\rho$, $\eta, \mathrm{P}_{\mathrm{B}} / \mathrm{N}_{0}$ and $\mathrm{R}$. Other simulation parameters are listed in Table 1 .

Table 1. Simulation parameters

\begin{tabular}{lll}
\hline Symbol & Name & Values \\
\hline$\eta$ & Energy harvesting efficiency & 0.7 \\
\hline$\lambda_{b s}$ & Mean of $\left|h_{b s}\right|^{2}$ & 0.5 \\
\hline$\lambda_{r r}$ & Mean of $\left|h_{r r}\right|^{2}$ & 0.5 \\
\hline$\lambda_{s r}$ & Mean of $\left|h_{s r}\right|^{2}$ & 0.5 \\
\hline$\lambda_{r d}$ & Mean of $\left|h_{r d}\right|^{2}$ & 0.5 \\
\hline$\gamma_{t h}$ & SNR threshold & 7 \\
\hline $\mathrm{P}_{\mathrm{B}} / \mathrm{N}_{0}$ & Beacon power to noise ratio & $0-30 \mathrm{~dB}$ \\
\hline $\mathrm{R}$ & Source rate & $3 \mathrm{bit} / \mathrm{s} / \mathrm{Hz}$ \\
\hline
\end{tabular}

Figs. 2 and 3 present the outage probability and system throughput versus $\rho$. In Fig. 2 and 3 , we set $\mathrm{P}_{\mathrm{B}} / \mathrm{N}_{0}$ at 5 , and 15 $\mathrm{dB}$, respectively and $\rho$ varies from 0 to 1 . The research results show that the outage probability has an increase when $\rho$ increase from 0 to 1 (Fig. 2). Moreover, Fig. 4 shows that the throughput falls $\rho$ increases from 0 to 1. In both Fig. 2 and 3, the analytical and simulation results agree very well with each other.

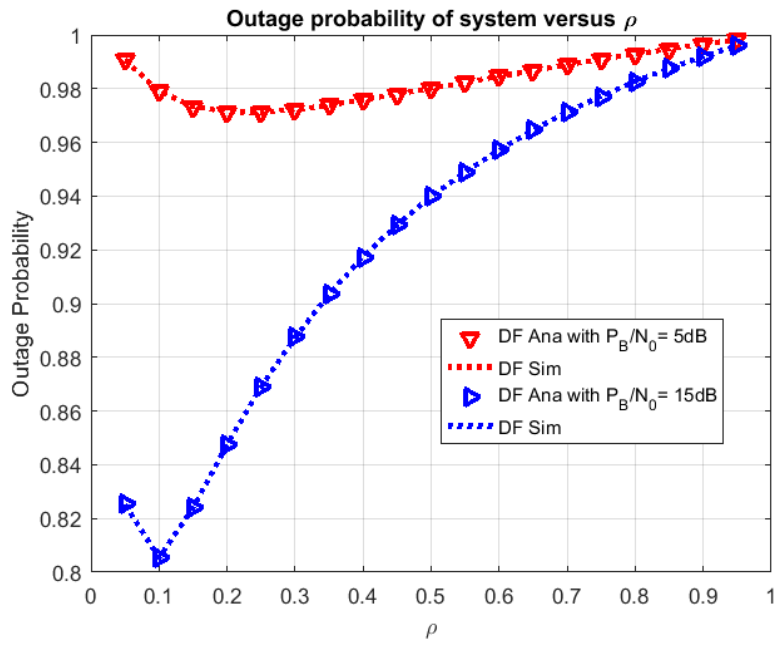

Fig. 2. Outage probability versus $\rho$. 


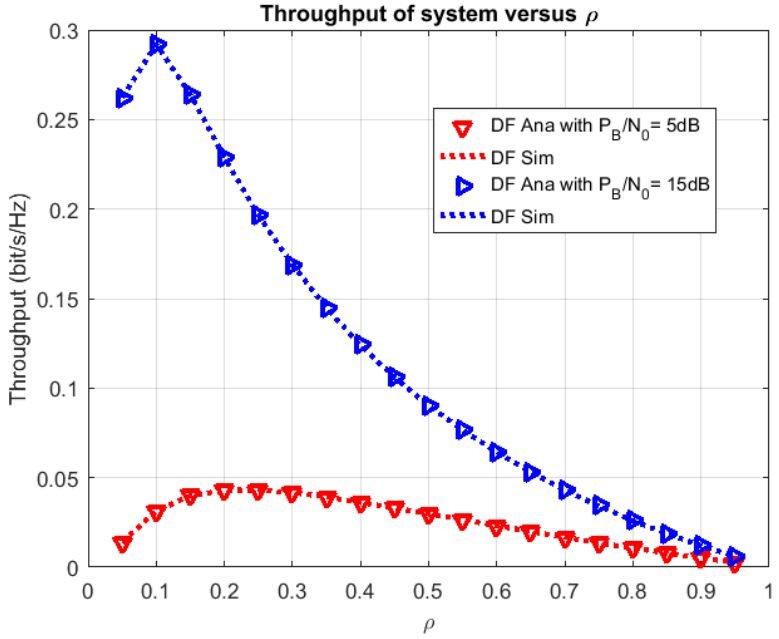

Fig. 3. Throughput versus $\rho$.

Furthermore, Fig. 4 and 5 show the effect of $\eta$ on the outage probability and system throughput with $\rho=0.3 ; 0.7$, and $P_{B} / N_{0}$ at 10 . The outage probability decreases while $\eta$ increases from 0 to 1 as shown in Fig. 4. In another way, the system throughput has a considerable improvement when $\eta$ increases from 0 to 1 as shown in Fig. 5. For both cases, the analytical analysis and the Monte Carlo simulation results are the same for all $\eta$ values.

Moreover, the outage probability and system throughput versus $\mathrm{R}$ is shown in Fig. 6 and 7, respectively. Similarity, we set $\mathrm{P}_{\mathrm{B}} / \mathrm{N}_{0}$ at $5 ; 15 \mathrm{~dB}$ and $\rho$ at 0.5 . From Fig. 6 we see that the outage probability increases. In contrast, the system throughput decreases (Fig. 7). Then the Figs. 8 and 9 present the effect of the ratio $\mathrm{P}_{\mathrm{B}} / \mathrm{N}_{0}$ to the outage probability and system throughput. In addition, Fig. 10 shows the optimal power splitting factor of the proposed system at $\mathrm{R}=0.5$ and $2 \mathrm{bps}$. In all figs, the simulation and analytical results totally agree with each other.

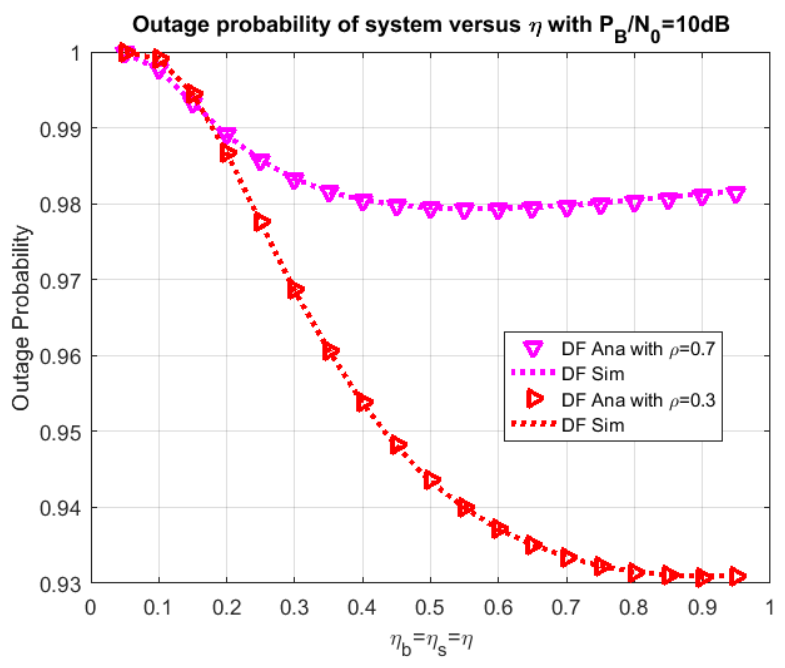

Fig. 4. Outage probability versus $\eta$.

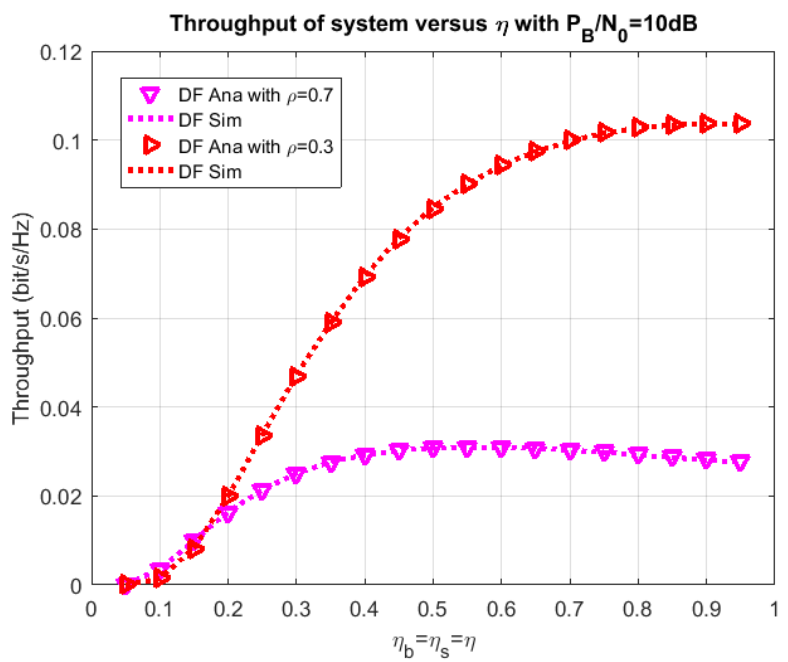

Fig. 5. Throughput versus $\eta$.

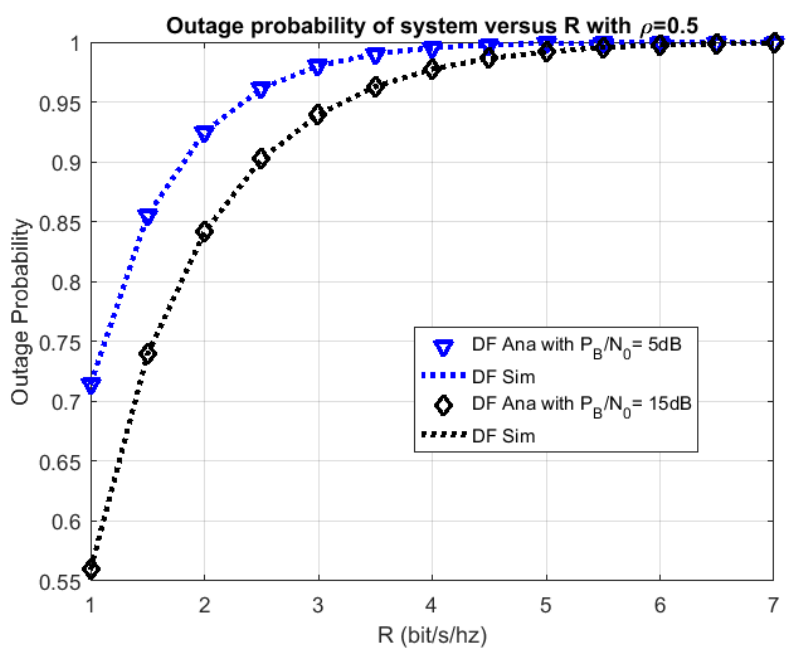

Fig. 6. Outage probability versus R.

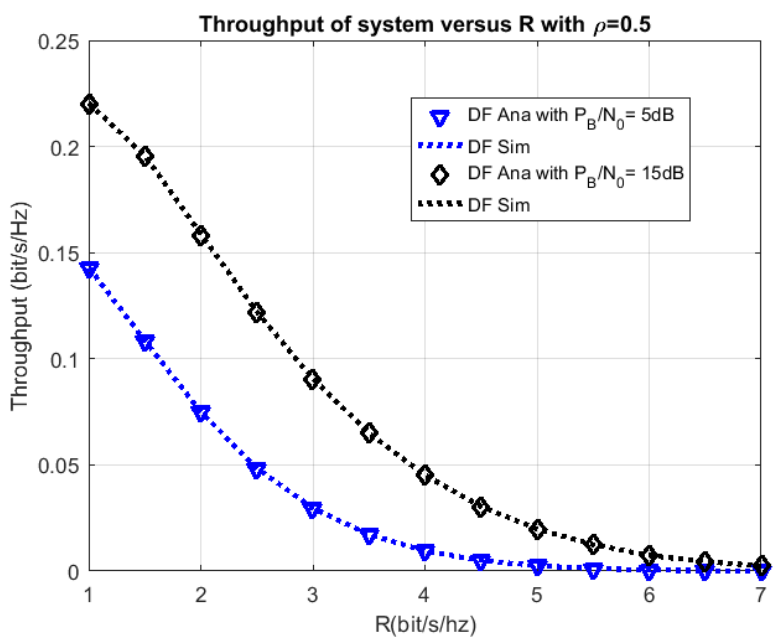

Fig. 7. Throughput versus R. 


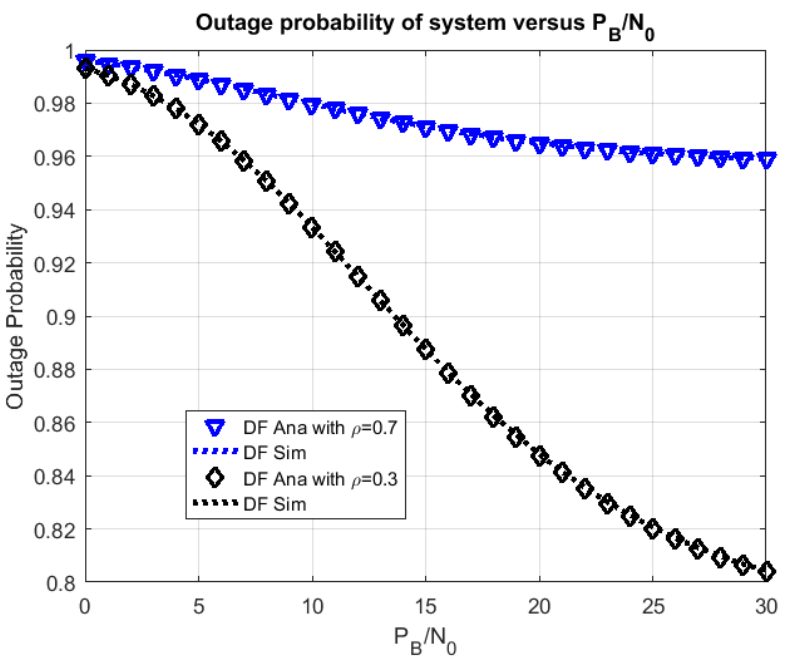

Fig. 8. Outage probability versus $\mathrm{P}_{\mathrm{B}} / \mathrm{N}_{0}$.

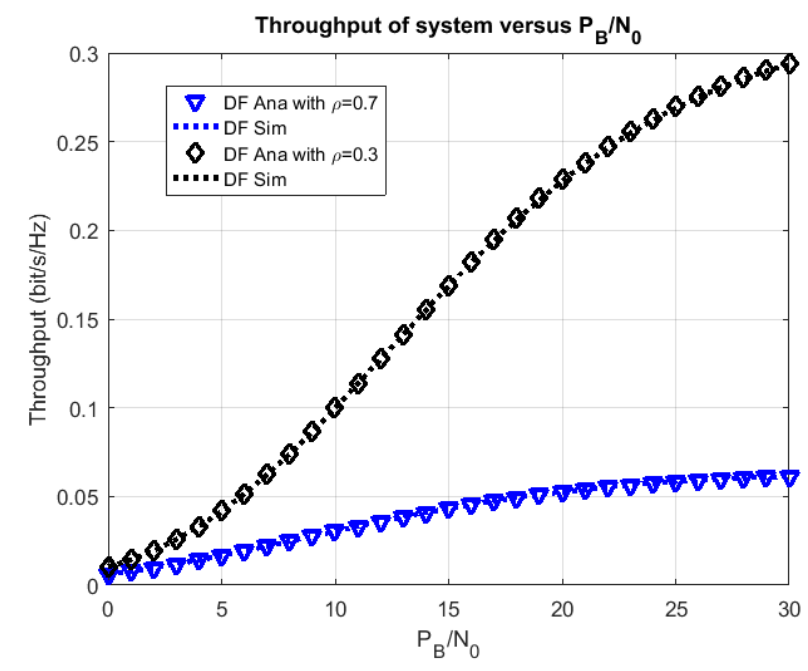

Fig. 9. Throughput versus $\mathrm{P}_{\mathrm{B}} / \mathrm{N}_{0}$.

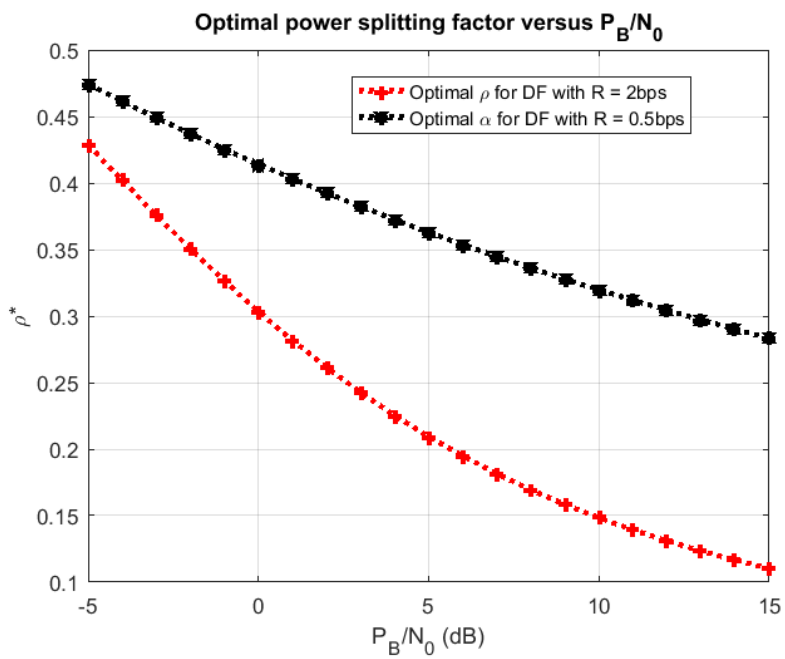

Fig. 10. Optimal time switching factor versus $\mathrm{P}_{\mathrm{B}} / \mathrm{N}_{0}$.

\section{CONCLUSIONS}

In this paper, we propose and investigate the full-duplex power beacon-assisted energy harvesting relay network with the power splitting protocol. For the system performance analysis, the closed-form expressions of the outage probability and system throughput are proposed and derived. Moreover, the analytical analysis is convinced entirely by the Monter Carlo simulation in connection with all system parameters. Furthermore, the optimal power splitting factor is presented and investigated. The research results show that the full-duplex power beacon-assisted energy harvesting relay network is the innovative relaying system for the communication relay network.

\section{ACKNOWLEDGMENT}

The research received financial support from the SGS grant No. SP2018/59, VSB-Technical University of Ostrava, Czech Republic.

\section{REFERENCES}

[1] F. De Rango, P. Lonetti, S. Marano, "MEA-DSR: A multipath energyaware routing protocol for wireless Ad Hoc Networks," IFIP International Federation for Information Processing, Vol. 265, pp. 215$225,2008$.

[2] H.-S. Nguyen, D.-T. Do, M. Voznak, "Two-way relaying networks in green communications for 5G: Optimal throughput and tradeoff between relay distance on power splitting-based and time switching-based relaying SWIPT," AEU - International Journal of Electronics and Communications, vol. 70, no. 12), pp. 1637-1644, 2016.

[3] M. Fotino, A. Gozzi, J,-C. Cano, C. Calafate, F. Rango, P. Manzoni, S. Marano, "Evaluating energy consumption of proactive and reactive routing protocols in a MANET," IFIP International Federation for Information Processing, Vol. 248, pp. 119-130, 2007.

[4] T.T. Phu, T.H. Dang, T.D. Tran, M. Voznak, "Analysis of probability of non-zero secrecy capacity for multi-hop networks in presence of hardware impairments over nakagami-m fading channels," Radioengineering, vol. 25, no. 4, pp. 774-782, 2016.

[5] T.N. Nguyen, T.T Duy, P.T. Tran, M. Voznak, "Performance evaluation of user selection protocols in random networks with energy harvesting and hardware impairments," Advances in Electrical and Electronic Engineering, vol. 14, no. 4, pp. 372-377, 2016.

[6] S. Bi, C. K. Ho, and R. Zhang, "Wireless powered communication: opportunities and challenges," IEEE Communications Magazine, vol. 53, no. 4, pp. 117-125, 2015.

[7] Y. Gu, H. Chen, Y. Li, and B. Vucetic, "An adaptive transmission protocol for wireless-powered cooperative communications," in proc. Of IEEE International Conference on Communications (ICC), 2015, art. no. 7248986, pp. 4223-4228.

[8] H. Ju and R. Zhang, "Throughput Maximization in Wireless Powered Communication Networks," IEEE Transactions on Wireless Communications, vol. 13, no. 1, pp. 418-428, 2014.

[9] K. Huang and V. K. N. Lau, "Enabling Wireless Power Transfer in Cellular Networks: Architecture, Modeling and Deployment," IEEE Transactions on Wireless Communications, vol. 13, no. 2, pp. 902-912, 2014.

[10] F. De Rango, M. Tropea, "Energy saving and load balancing in wireless ad hoc networks through ant-based routing," in proc. of International Symposium on Performance Evaluation of Computer and Telecommunication Systems 2009, SPECTS 2009, pp. 117-124.

[11] F. De Rango, M. Tropea, "Swarm intelligence based energy saving and load balancing in wireless ad hoc networks," in proc. of the 2009 Workshop on Bio-inspired Algorithms for Distributed Systems, BADS '09, pp. 77-83. 
[12] Y. Ma, H. Chen, Z. Lin, Y. Li, and B. Vucetic, "Distributed resource allocation for power beacon-assisted wireless-powered communications," in proc. of IEEE International Conference on Communications (ICC), 2015, art. no. 7248924, pp. 3849-3854.

[13] Tan N. Nguyen, T.H.Q.Minh, Phuong T. Tran and Miroslav Voznak,"Adaptive Energy Harvesting Relaying Protocol for Two-Way Half Duplex System Network over Rician Fading Channels", Wireless Communications and Mobile Computing, Vol.2018, 10 pages, Apr.2018.

[14] J.-H. Park, Y.-S. Jeon, and S. Han, "Energy Beamforming for Wireless Power Transfer in MISO Heterogeneous Network With Power Beacon," IEEE Communications Letters, vol. 21, no. 5, pp. 1163-1166, 2017.

[15] C. Zhong, S. Jin, K.-K. Wong, and M. R. Mckay, "Ergodic Mutual Information Analysis for Multi-Keyhole MIMO Channels," IEEE Transactions on Wireless Communications, vol. 10, no. 6, pp. 1754 1763, 2011.

[16] T. Q. Duong, T. T. Duy, M. Matthaiou, T. Tsiftsis, and G. K. Karagiannidis, "Cognitive cooperative networks in dual-hop asymmetric fading channels," in proc. of IEEE Global Communications Conference (GLOBECOM), 2013, art. no. 6831197, pp. 955-961.

[17] E. K. P. Chong and S. H. Zak, An introduction to optimization, $4^{\text {th }}$ ed., Wiley, 2013, pp. 1-640.

[18] Tan N. Nguyen, Phuong T. Tran, T.H.Q.Minh and Miroslav Voznak,"Two-Way Half Duplex Decode and Forward Relaying Network with Hardware Impairment over Rician Fading Channel: System Performance Analysis", Elektronika Ir Elektrotechnika,Vol.24, No.2, pp.74-78, Apr.2018 